\title{
The Study of Audience's Response to the Fake News About Covid-19 That Posted by KOL on Twitter
}

\author{
Zewei Jin ${ }^{1,{ }^{*}, \dagger}$, Nuo Mei $^{2, *, \dagger}$, Yixuan Wang ${ }^{3, *, \dagger}$ Yunlong Zhang ${ }^{4, *}{ }^{*}$, , \\ ${ }^{1}$ University of Toronto St. George Campus, Toronto, M5S 2E8, Canada \\ ${ }^{2}$ University of Toronto Mississauga Campus, Mississauga, L5L 1C6, Canada \\ ${ }^{3}$ University of Toronto St. George Campus, Toronto, M5S 2E8, Canada \\ ${ }^{4}$ Communication University of China, No.7, Dingfuzhuang South Li, Chaoyang District, Beijing, P.R. China \\ *Corresponding author.Email:nuo.mei@mail.utoronto.ca \\ Those authors contributed equally.
}

\begin{abstract}
In the current era of social media culture, almost everyone's social life is inseparable from media. Especially since the outbreak of COVID-19, the way most people communicate has shifted to online, and the release of information on social media has seen an explosion. However, the information spread on social media platforms is not all positive, and there is many fake news mixed in and continuously spread. Different types of publishers have different effects on their audiences, and their audiences react differently. This paper uses thematic analysis and selects the content of a fake news tweet published by a journalist, and studies the content of its popular comments to summarize the audience's response. The results turned out that the responses of its audience fall into three broad themes: unequivocal belief, decisive denial, and skepticism.
\end{abstract}

Keywords: social media, Twitter, fake news, covid-19.

\section{INTRODUCTION}

Social media is multimodal and is of different types, functioning to rapidly spread news and information in a way that changes how 'information is passed across societies and around the world' [1]. Besides having the ability to transmit news from verified sources, social media can shape the way that people connect with others and judge the veracity of the information they are exposed to.

During the COVID-19 pandemic, people's work, study, and life changed a lot, with many activities transferred online and most communication limited to online platforms as people became physically isolated. A CNBC report showed rapid growth in Twitter's user activity and profit during the pandemic, with users' confidence in the platform increasing the more they used it [2]. Twitter incorporates aspects of social networking sites such as Myspace and Facebook, with instant messaging technologies creating networks of users who communicate throughout the day with brief "tweets." Twitter data scientist Douglas Mason analyzed over 2 million tweets sent by thousands of verified users over the course of one month and found that adding video, links, and photos impressively boosts the number of retweets, regardless of the factual content. Organizations intending to mislead the public for whatever motive can use these methods to rapidly disseminate misinformation that does not appear suspicious or questionable. When such messages are picked up by celebrities, who already have enormous numbers of followers who are likely to believe their words or recommendations, the celebrities may, with the best of intentions, quickly retweet it without checking facts, effectively endorsing it by taking it seriously or commenting on it with approval.

Although the expression "fake news" was popularized during the US elections in 2016 as a way to discredit traditional news media, it and other phrases such as "fabricated news" are commonly used to describe rumors and false news stories spread through social media, either maliciously or carelessly [3]. "Fake news primarily denoted inaccurate news pieces, which were often intentionally fabricated ... [and] circulated via social media" [3]. Whether generated for a political or economic purpose or the by-product of misunderstanding or fantasy, fake news is good at disguising itself to "strategically exploit existing and trustworthy 
information channels as a cloak to disseminate their messages." [3]

Throughout the pandemic, there has been a deluge of fake news, conspiracy theories, and misinformation, making it difficult for platforms and users to distinguish the authenticity of messages. On Twitter particularly, with countless short and frequent tweets that can instantly be retweeted to thousands, having a lingering effect even if they are later removed after being false, fake news can have vastly negative effects on a population. An anthropological study shows that when people have little control over environmental threats, unverified news will thrive. According to an Editorial in the Lancet [4], too many news sources form an infodemic, leaving people less likely to find useful and reliable information. Even in the cases where the information is from medical or scientific sources, if it is spread to non-experts before being vetted (as in preprints of articles that have not yet been peer-reviewed and revised accordingly), it can undermine public trust by offering conflicting views and findings that laypeople cannot accurately judge. Consequently, people overlook or even distrust news from trustworthy authorities, blocking cooperation and communication [4].

\subsection{Literature Review}

Fake news is the news that appeals like real news, but they contain false information. Misinformation has been with us since the development of the earliest writing system. As a result, people set various laws to deal with it [1]. While digitalization has challenged the traditional model thoroughly. Social media is a very powerful medium with a large number of non-supervised content, which means it could empower the misinformation phenomenon and even manipulate public opinion [2]. And the overabundance of information has formed an infordemic during the covid-19 pandemic [3]. Despite the hard work paid off by fact-checking sites and regulation departments, there is still much fake news wild spread on social media, and some of them could be fatal. For example, vaccine conspiracy would reduce vaccination intentions by inducing undue concern and increasing powerlessness, disillusionment, and mistrust [4]. People fabricate misinformation mainly for financial gain, political gain, and experimental manipulation [5]. People share fake news because they formed a world view in a partisan and polarized way. And fake news is a part of the larger media ecosystem. And the platform also contributes to this. The algorithms and ad systems promote or incentivize problematic content [6]. Some scholars also used the affordance theory and cognitive load theory to explain fake news sharing. Najmul Islam et al. find that different propose of social media usage would bring problematic consequences, like increased fake news spreading [7]. Moreover, the big companies that manipulate social media determine that social media is a significant part of spreading fake news [8]. Bell Emily et al. stated that fake news on social media is easily spread by people who are highly engaged in social media [8]. The companies which manipulate the social media platform are not merely technical companies. These companies are the new platform. The purpose of these companies is to attract more attention from people. As a result, the content in the social media platform is not neutral, as Bell Emily et al. noted that these companies' business models had prompted the spread of fake news in the social media platform [8]. Therefore, social media accounts are also a hotbed of fake news. The organization account is a kind of brand [9]. As Gautam Kishore Shahi noted, the organization's communication approach is more professional, attracting more users' attention [9].

In addition to organizational accounts, another type of account that is highly involved in social media conversation and has a huge impact is the personal accounts of famous people. This category includes entertainment celebrities, political celebrities, Internet celebrities, and authority figures who have succeeded in certain fields. A strong fan base characterizes this type of account, and once they post information, a large number of viewers like, comment, and retweet it. According to the journal article of William Brown, he analysis four stages of the audience that are involved with media persona [10]. The literature illustrates that media personalities, whether virtual or real, can be powerful information agents on social media. They have a powerful influence on social change. The relationship between them and their fans includes information transportation, parasocial relationships, identification, and worship. From shallow to deep, these four relationships illustrate how convincing fans are to the media figures they follow and prove that these media figures have a huge influence on the public, especially fans. Another study, written by Kumju Hwang and Qi Zhang, looked at celebrity benefits and followers' desire to buy the products they endorse. The presence of quasisocial relationships will increase the effectiveness of celebrity influence and their persuasiveness to their fans [11]. It can be seen that the information released by celebrities' social media account will be widely viewed and spread. Their information is more credible and persuasive than other private accounts. As a result, the negative impact of fake news published by celebrities will be doubled or more. This article will analyze and discuss the negative impact of fake news published by celebrities on Twitter, and study how to monitor and solve the spread of fake news on social media in the future.

\section{METHODOLOGY}

The research method the team chooses is content analysis in qualitative research. Through content analysis, the paper will focus on the details and discover the influence of fake news on people. Based on the paper's 
topic, the team finds fake news that was posted by a celebrity, whose name is Nick Hinton, on Twitter. The research will be based on the number of likes, comments, and retweets of the celebrity's post, which is fake. This paper collected all content on social media on August 15, 2021. Four team members participated in the information collection. This team searched for the keywords "Twitter", "Fake news", "COVID-19," and "Celebrity" and found a British artist who matched the topic of the research. On February 15, 2020, an artist, author, and podcaster named Nick Hinton posted a tweet referring to the covid-19 that was predicted back in 1981, accompanied by a screenshot of a page in the book that mentions the word "Wuhan-400".The celebrity has 116.6k followers on Twitter, and as of the search date, the post had 6,078 likes, 3,213 retweets, and 862 quotes.

The study adopts the thematic analysis method to analyze the retweets sample. After detected 50 retweet messages, we find that there about four main themes among these retweets textual. All 50 texts could be separated into "fact-checking", "conspiracy", "entertaining", and non-sense. We followed two indexes to induct these texts: belief or not and whether the author takes it seriously. If a user retweet that he/she believes the tweet is in a quite serious mood, it means the author believes the conspiracy theory that some group planned the coronavirus. If the tweet includes some fact-checking materials, it shows that the user who retweets is very serious, but they do not believe the tweet at all. For the people who traits the prophecy unserious, the tweet is just entertainment for them. It is not important whether they believe it or not, but it is fun or new. Apart from these themes, some people retweet something irrelevant to the topic.

Table 1. Themes classification

\begin{tabular}{lll}
\hline & Belief & Not belief \\
\hline Serious & conspiracy & Fact-checking \\
Not serious & entertaining & Non-sense
\end{tabular}

The Twitter account is a public account of Nick Hinton, not his private account. Therefore, the post from Nick Hinton that we choose, which is also publicly available. Thus, there is no copyright issue. Moreover, all of the research and analysis will be on account of facts and data. The study will not be emotional and subjective.

\section{RESULTS AND DISCUSSION}

On February 16th, 2020, Nick Hinton, an influential Twitter user, uploaded a tweet. It announced that Dean Koontz had predicted the outbreak of coronavirus in his novel The Eye of Darkness. He attached two photos to this tweet. The one is the cover of Koontz's The Eye of Darkness. The other one is one page in that book. Nick circled the Wuhan-400 in the book and underlined a statement that said the virus was created outside Wuhan's RDNA labs. This page also tells many details of the socalled Wuhan- 400 virus. The truth is, until now, there is no evidence show that coronavirus is a human make virus, as well as how it appears. And in the first published version of the novel, the virus was called Gorkij-400, named after a Russian town. And in the later version, it changed its name to Wuhan for some reason. The detail of the virus also shows a considerable difference between coronavirus. Still, this tweet has received 471 comments, 3211 retweets, 863 quotes, and 6082 likes until August 21 st. As its comments show, people all over the world retweeted this fake news in various languages. Some commenters show a favourable attitude toward this tweet. They strongly believe it. In contrast, some people pointed out that this is fake, even post some evidence proof link below.

In this study, the team members selected the top 50 popular comments under Twitter for multiple analyses. However, there is no clear directivity result (see appendix1), so the discussion of this study will mainly focus on the content research of the two most popular comments.

There is about one-fifth of people do not trait the tweet seriously. Despite some of them do believe that Koontz manages to predict the outbreak somehow. Compared to the people who believe conspiracy theory and ask to move, these people just acknowledge an interesting thing. They do not worry about the conspiracy, even the truth. All they need is a sense of fulfilment when they add something interesting things up to the tweet. As Neil Postman said, we are entering an entertainment age. Items become lost their meaning when we do not trait them well. The amusement function is the only valuable thing left to the audience of mass media.

For example, Aden Bead retweeted Nick Hinton on February 16, that the lab opened in 1958, and the book was published in 1981 with a dynamic emoticon. A young Latino uses the judger's hummer to knock the table and say something with a big smile in his room. There are two words on the screen "next case." It shows that the user who retweets the conspiracy-like tweet is in a relaxed mood, despite the belief that there are some connections between the book and the virus lab, unlike others who ask to do something. After he checks the information and makes a quick conclusion, he chooses to skip it and move to the next case, like the emoticon shows. It is a very contradictory situation that if he agrees that the virus is human-made, it ought to be a trying situation, but he just ignored it and moved to the next attraction.

By analyzing different variable, the data are split into different categories of keywords. The one that interacted the most is a conspiracy. By searching and rearranging the comment, the top comment is by Mukhtar Qule (@MuktarAQule), which received the most likes from 
490 audiences, 50 replies, and 108 retweets. The comment is in the direction of playing with calculations by combining numbers in a way to "support" the conspiracy theory that Koontz predicted a man-made virus coming from Wuhan in his 1981 novel, which Nick Hinton tweeted with picture evidence. From Mukhtar's comment, along with his replies to some of the others who replied to his first tweet, it is apparent that he believed in the conspiracy theory and was not merely playing along as a joke: "it is a conspiracy theory and takes your part if you are interested otherwise leave it", "Thanks. You got another theoretical assumption to prove this conspiracy theory about the facts of the CORONOVIRUS." Although several of the replies were meant as criticisms or corrections, other replies and the many likes and retweets show that his tweet sustained interest in the theory, with many people agreeing with his comment. Comments like "It's a man-made virus", "Final that's it. It was made. Coronavirus is a group of virus put together ...", "Nice points", "This calculations are shocking", "I like your math" all show support and some level of belief. Although Mukhtar's tweets before and after the Hinton thread were mainly about politics or regional 7., activity in Somaliland, along with some pro-Trump, anti-China, anti-gay, and pro-Muslim messages, there is no pattern of support for other conspiracy theories, replies to Hinton, or numerology, so this particular thread may just have been related to his political beliefs. His purpose was likely to help sustain the "made in a Wuhan lab" theory because that is consistent with his tweets about China. Taiwan is one of the few regions to recognize Somaliland sovereignty. The impact of this tweet on others is surprisingly large, considering Mukhtar's few followers and lack of any other connection to the topic in his tweets.

The second theme is fact-checking. The comment in this part is the second most popular comment. The content of the comment is completely different from the first one. This comment is more focused on the book's facts. The replier did not trust Nick Hinton easily but researched the book's actual content. The second comment was from Mell@ttatini. The comment posted by Mell@ttatini focused on the real content of book, and Mell@ttatini did the fact research. Mell@ttatini did not believe Nick Hinton's post, and Mell@ttatini found that the pictures in Nick Hinton's post are different from the book's original content. Thus, Mell@ttatiniell added the picture of what she found in the book in the comment. In addition, Mell@ttatini's comment has 186 likes, 78 comments, and 35 retweets. The people who comment on Mell@ttatini's comment can be categorized into three groups. The first group of people is those who did not pay attention to the content of the picture that Mell@ttatini posted but are more concerned with the language of the picture. These people think that Gorki 400 in the book is Wuhan 400 that Nick Hinton posted. The reason is that these people found the language of the picture that Mell@ttatini posted is not English. Hence, they thought if they translated Gorki 400 into English, the meaning of Gorki 400 in Wuhan 400. Thus, these people who were concerned about the language of the book essentially believed what Nick Hinton had posted, even though they browsed the picture that Mell@ttatini added in the comments. The second group of people is those who are concerned about the timing of the book's release. They think that the book Mell@ttatini read is not the latest edition, and they believe that Gorki 400 in Wuhan 400 is the new edition of the book. Therefore, they also believed Nick Hinton was correct. The last group of people just occupied a small number. It seemed that they believed Mell@ttatin. These people commented that they wanted a PDF version of the book that Mell@ttatin posted in the comment. In conclusion, based on the research of the second popular comment on Nick Hinton's post, it can be obviously found that Mell@ttatin's comment made almost no impact on the viewers; people are more likely to trust Nick Hinton who has more influence. As a result, although Mell@ttatini added the real content in the comment, people still were not influenced by the comment of Mell@ttatini.

\section{CONCLUSION}

This paper mainly studied how the audience responded to the fake news posted by KOL on Twitter. The study group selected a fake news post about "COVID-19 was predicted in a book" by a well-known journalist on February 16, 2020, to discuss audience reactions to the tweet. Based on the 50 samples, the themes of audience responses were broadly divided into three categories: complete belief, decisive rejection, and indifference. The most representative comments on each topic were selected for research. The diversity of topics in the comment section of this Tweet makes it possible to lock in the direction of future research on why the audience has many different topics of comments and whether the theme of comments is different due to different classes, education background, and growth environment.

\section{REFERENCES}

[1] Mayfield, Thomas D., A Commander's Strategy for Social Media. NDU Press.

[2] Novet, J. (2021, July 23). Twitter posts fastest revenue growth since 2014 in pandemic rebound. CNBC. https://www.cnbc.com/2021/07/22/twittertwtr-earnings-q2-2021.html

[3] Quandt, Thorsten et al. (April 2019) Fake News. University of Münster, Germany, pp.1-6, 10.1002/9781118841570.iejs0128.

[4] Zarocostas, J. (2020). How to fight an infodemic. The Lancet, 395(10225) https://doi.org/10.1016/s0140-6736(20)30461-x 
[5] Tandoc Jr, Edson C., Zheng Wei Lim, and Richard Ling. "Defining "fake news" A typology of scholarly definitions." Digital Journalism 6.2 (2018): 137-153.

[6] Ireton, Cherilyn, and Julie Posetti. Journalism, fake news \& disinformation: a handbook for journalism education and training. Unesco Publishing, 2018.

[7] Diseases, The Lancet Infectious. "The COVID-19 infodemic." The Lancet. Infectious Diseases 20.8 (2020): 875.

[8] Jolley, Daniel, and Karen M. Douglas. "The effects of anti-vaccine conspiracy theories on vaccination intentions." PloS one 9.2 (2014): e89177.

[9] Diseases, The Lancet Infectious. "The COVID-19 infodemic." The Lancet. Infectious Diseases 20.8 (2020): 875.

[10] Marwick, Alice E. "Why do people share fake news? A sociotechnical model of media effects." Georgetown Law Technology Review 2.2 (2018): 474-512.

[11] Islam, A K M Najmul et al. "Misinformation sharing and social media fatigue during COVID-19: An affordance and cognitive load perspective." Technological forecasting and social change vol. 159 (2020): 120201 . doi: 10.1016/j.techfore.2020.120201

[12] Emily, Bell et al. "Introduction: Watermelons to democracy." The Platform Press How Silicon Valley Reengineered Journalism 1.1, (1970): 13-16.

[13] Shahi, Gautan Kishore. "An exploratory study of COVID-19 misinformation on Twitter." 5.8, (2021): $1-16$.

[14] Brown, William J. "Examining Four Processes of Audience Involvement With Media Personae: Transportation, Parasocial Interaction, Identification, and Worship." Communication theory, 2015-08, Vol.25 (3), p.259-283.

[15] Hwang, Kumju ; Zhang, Qi. Influence of parasocial relationship between digital celebrities and their followers on followers' purchase and electronic word-of-mouth intentions, and persuasion knowledge. Computers in human behavior, 2018-10, Vol.87, p.155-173 